\title{
EXCAVACIONES EN ALERO GIANELLA, CURSO MEDIO DEL VALLE DE CHACABUCO (ANDES CENTRO PATAGÓNICOS)
}

\author{
FELIPE FUENTES-MUCHERL', FRANCISCO MENA", JOSÉ BLANCO"**" Y CATALINA CONTRERAS"*m"
}

\begin{abstract}
RESUMEN
El presente estudio aborda la excavación y análisis zooarqueológico y lítico de un pequeño alero con arte rupestre, ubicado en el curso medio del valle de Chacabuco (Patagonia central). Aunque el sitio y sus pictografías son conocidos desde hace años, su excavación permitió asociarlo con eventos de caza estival. La preparación y uso de variados artefactos líticos permite sospechar, sin embargo, de otras funciones dentro de visitas en un período restringido a unos pocos siglos. Una fecha de $2720 \pm 25 \mathrm{AP}$ sugiere además que esta zona fue ocupada más regularmente y antes de lo que se creía, y aunque se confirma la relación con zonas orientales complementariamente al aprovechamiento de materias primas líticas locales.
\end{abstract}

PALABRAS CLAVE: Patagonia, cazadores-recolectores, río Chacabuco, zooarqueología, sistema de asentamiento.

\section{EXCAVATIONS AT ALERO GIANELLA, MIDDLE CHACABUCO VALLEY (CENTRAL PATAGONIAN ANDES)}

\footnotetext{
ABSTRACT

The present study addresses the excavation and faunal and lithic analysis of one small rock shelter with rock paintings on an Andean valley in South-Central Patagonia. Although the zooarchaeological context is largely concordant with summer hunting forays, the preparation and use of diverse lithic artefacts suggests other activities were performed at the site, besides its use as a hunting stand. A $2720 \pm 25 \mathrm{BP}$ radiocarbon date at the base indicates also that this area was occupied earlier and perhaps more regularly than previously thought. Although the relationship with the eastern plains is confirmed, we observe also the use of local raw materials.

KEY WORDS: Patagonia, hunter-gatherers, Chacabuco river, zooarchaeology, settlement system.

Argomedo 390, depto 2102, Santiago, Chile. felipe.fuentes.mucherl@gmail.com.

* Centro de Investigación en Ecosistemas de la Patagonia (CIEP) I. Serrano 509, Coyhaique, Chile. francisco.mena@ciep.cl.

** Universidad Nacional del Centro de la Provincia de Buenos Aires (UNICEN), Avenida Del Valle 5337, Olavarría, Argentina. jfblanco@gmail.com.

*s** catalina.contreras.mira@gmail.com.
} 


\section{INTRODUCCIÓN}

Recientes estudios en el valle del río Chacabuco (Aisén, Chile), han revelado interesantes diferencias con otras regiones estudiadas en Patagonia central, tanto en Chile como en Argentina (Lucero y Mena 2001, Aschero et al. 2007). Por ejemplo, la zona expone una ausencia de fechas tempranas, y una mínima presencia de pinturas rupestres en relación a valles circundantes.

El presente trabajo se centra en el estudio de uno de los pocos sitios conocidos con arte rupestre en el valle, entregando datos cronológicos, e información a propósito del sistema de asentamiento, subsistencia y tecnología lítica por parte de estos cazadores-recolectores.

El sitio Alero Gianella se ubica al sur del lago Gutiérrez (474'47.97" lat. S y $72^{\circ} 16^{\prime} 20.31^{\prime \prime}$ long. O). El alero se orienta hacia el norte, ocupando parte de una amplia pared rocosa, desde donde es posible visualizar el lago, y los distintos corredores que conectan con el curso medio del río Chacabuco (Fig. 1). El sitio, adicionalmente se encuentra adyacente a dos pisos vegetacionales importantes, representados en su parte alta por bosques y en su sección baja por estepa, extendiéndose esta última hasta las terrazas del lago Gutiérrez, todo lo anterior asociado a una extensa estación fría. Cabe mencionar también, que el sitio fue descubierto inicialmente por F. Bate (1970), y posteriormente vuelto a identificar durante la primera campaña de prospección del presente proyecto, bajo la denominación inicial de $\mathrm{XI} / 2$ (Abril 2009).

\section{MATERIAL Y MÉTODO}

Se excavaron dos unidades de $2 \times 2 \mathrm{~m}$, siguiendo la estratigrafía natural, en complemento al constante registro de perfiles y plantas. Los sedimentos fueron cernidos en un harnero con grilla de $2 \mathrm{~mm}^{2}$. Todos los hallazgos de $2 \mathrm{~cm}$ o más, fueron registrados tridimensionalmente.

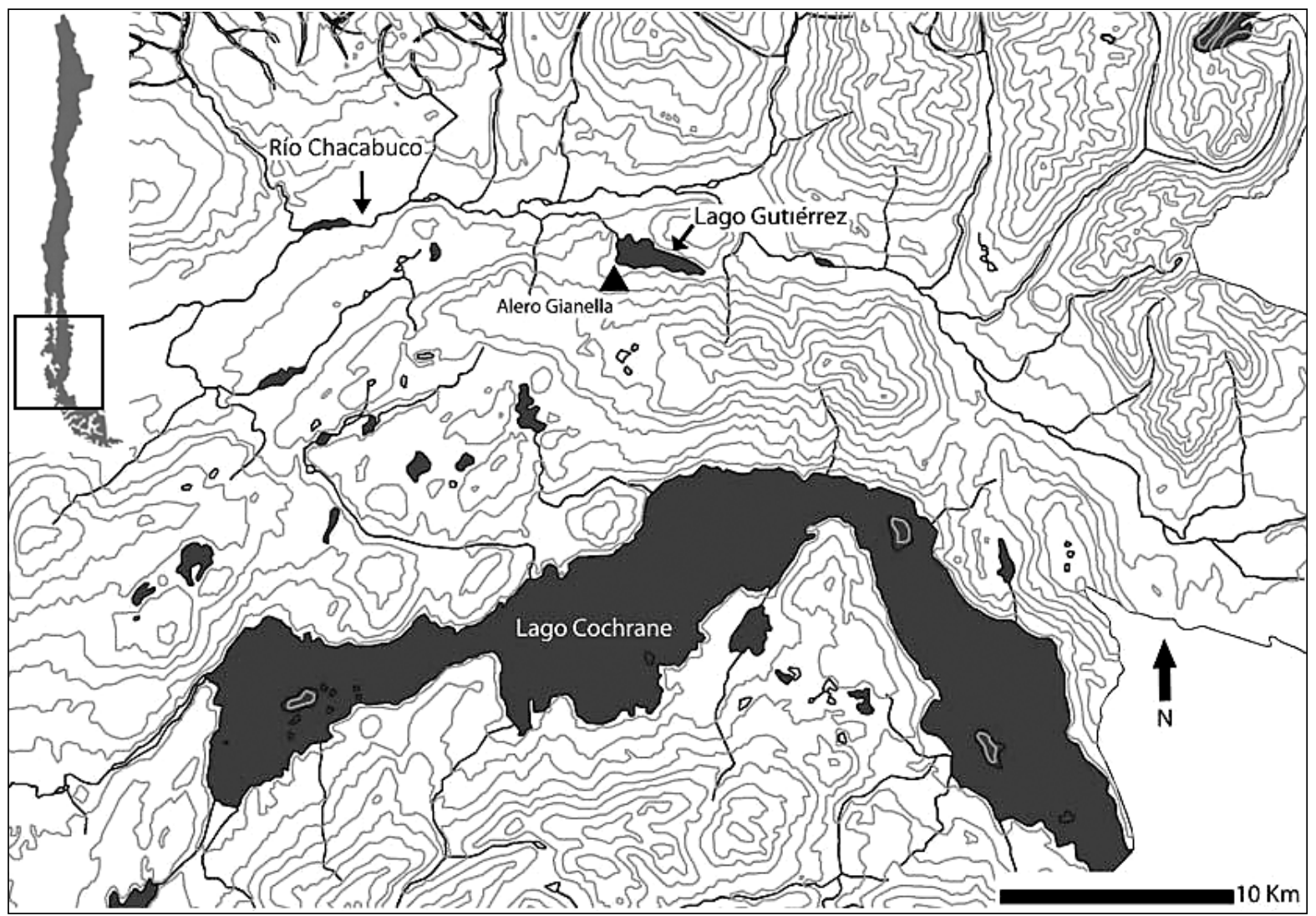

Fig. 1. Ubicación del sitio Alero Gianella. 

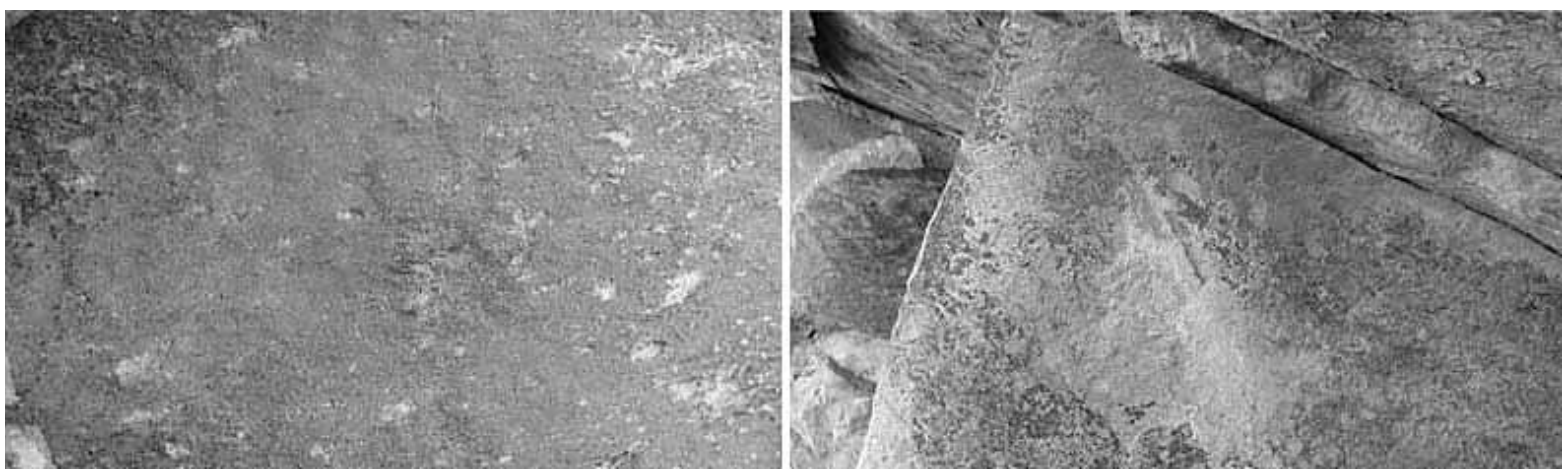

Fig. 2. Arte rupestre en Alero Gianella, con detalle de soporte izquierdo y derecho.

Para el análisis arqueofaunístico, se utilizó esqueletos de referencia y manuales osteológicos (Altamirano 1983; Pacheco et al. 1986). Se consideraron además las improntas dejadas por agentes naturales y antrópicos, aludidas en la bibliografía especializada (cf. Binford 1981; Lyman 1994).

La estimación de la densidad mineral ósea, específicamente para el caso de los camélidos y cérvidos, se realizó tomando en cuenta los valores propuestos por Elkin (1995) y Lyman (1984). Mientras que para la utilidad económica fueron considerados los índices existentes para guanaco y huemul, definidos en Borrero (1990) y Belardi y Gómez (1998). Con la finalidad de medir la intensidad del aprovechamiento cárneo-medular, la longitud de los fragmentos diafisiarios fue medida (Mengoni y de Nigris 1999).

Por otro lado, el análisis lítico contempló variables nominales, de intervalo y métricas, las que fueron escogidas en función de estudios anteriores (Méndez y Blanco 2001; Méndez et al. 2004).

\section{EXCAVACIÓN EN ALERO GIANELLA}

Al este de la grilla de cuadriculas se registró el negativo de dos manos izquierdas, a las que se suma parte del antebrazo, las que fueron realizadas sobre una pronunciada inflexión del paredón, permitiendo la oposición de los motivos (Figs. 2 y 3).

Con posterioridad al registro de arte rupestre, se implementaron dos unidades, las que permitieron determinar la existencia de cerca de ocho capas estratigráficas (Figs. 3 y 4).

De la unidad A1, específicamente de la base de la capa 5, se obtuvo un fechado AMS, a partir de carbón vegetal, con resultado $2710 \pm 50$ AP (UGAMS 5867), el que fue calibrado con dos sigmas (programa OXCAL 4.0), permitiendo ubicar la muestra en un rango que va del 969 al $670 \mathrm{aC}$ (Fig. 4).

El análisis de las distribuciones líticas y óseas, desprendidas del registro en planta, exponen una distribución preferentemente mediada por las condiciones del terreno y agentes naturales (Fig. 3), con

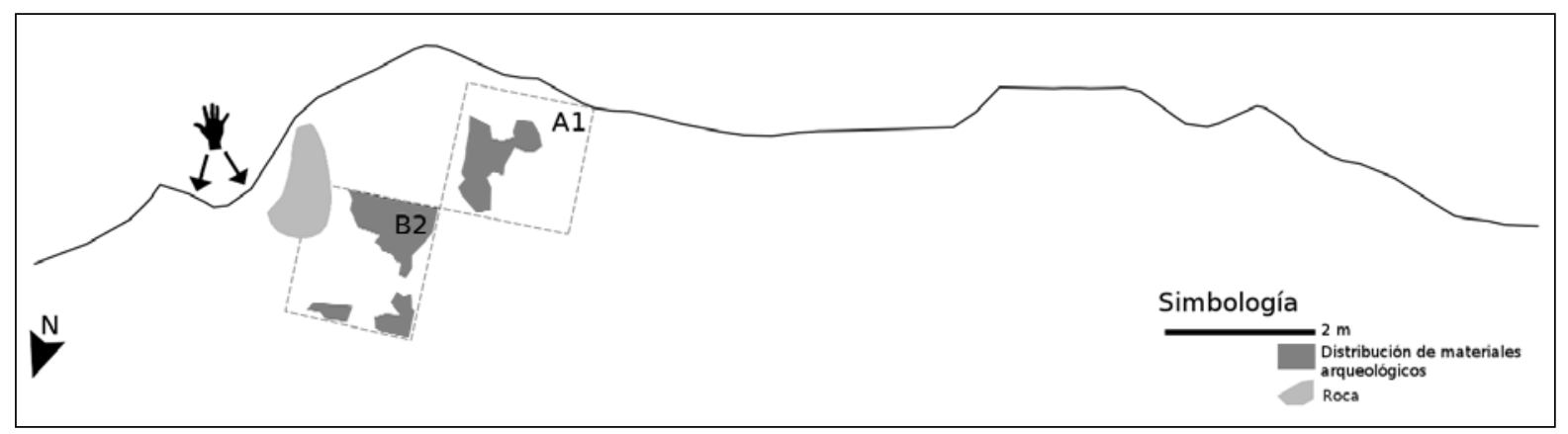

Fig. 3. Planta del sitio Alero Gianella, con ubicación de unidades y área con manifestaciones rupestres. 


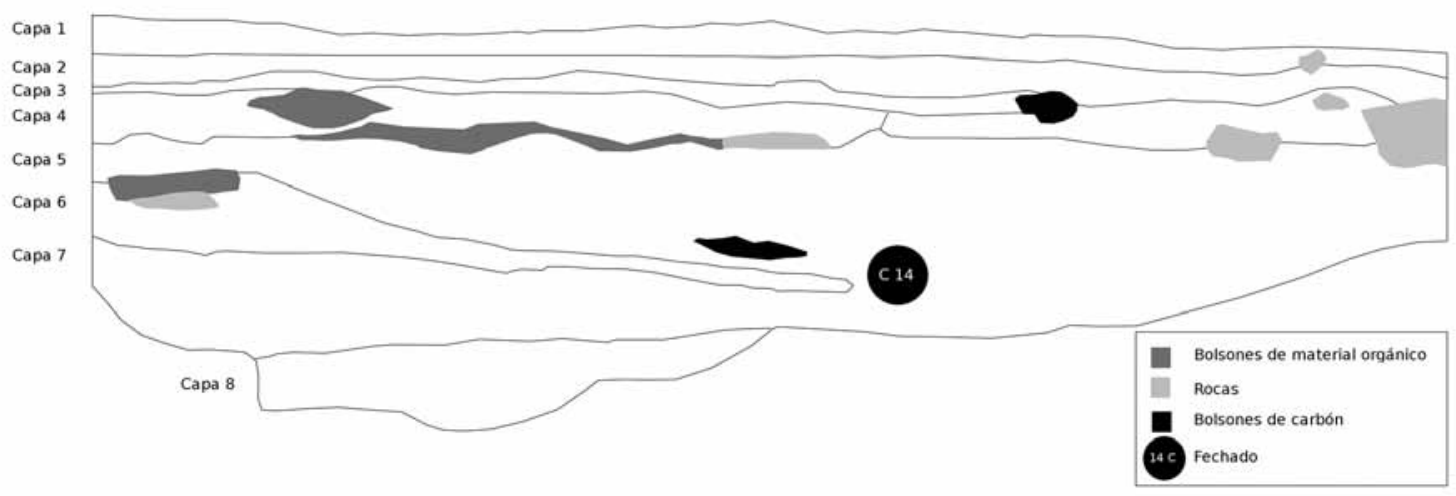

Fig. 4. Perfil estratigráfico del sitio Alero Gianella; Capa 1. Guano suelto; Capa 2. Guano compacto; Capa 3. Roca desintegrada; Capa 4. Sedimento muy orgánico asociado a raíces; Capa 5. Área con abundantes espículas y lentes de quema; Capa 6. Sedimento muy orgánico similar a capa 4; Capa 7. Sedimento arcilloso y muy compacto; Capa 8 . Sedimento arcilloso de compactación variable, similar a capa 7.

una mayor representación de elementos en la capa 5, desde donde algunos especímenes habrían migrado verticalmente hacia la superficie y la base. La causa de tal dispersión deriva de roedores, identificados por abundantes restos esqueletales y la presencia de túneles y madrigueras.

Las dispersiones horizontales, en tanto, se encuentran condicionadas por grandes bloques, que ocupan el flanco este de la unidad B2, sumándose posibles factores gravitacionales, los que aglutinarían las piezas hacia los vértices que unen ambas unidades. No se descarta la acción de roedores en la dispersión horizontal de los restos.

\section{ARQUEOFAUNA Y LÍTICA DE ALERO GIANELLA}

Se identificaron 747 especímenes óseos, de los cuales sólo 80 han podido ser determinados a especie, mientras que los siguientes fueron adscritos a artiodactyla generalizado (cérvidos y camélidos) y Chloephaga sp. (gansos). Se excluyen del presente trabajo los restos de roedores, los cuales son intrusivos y post-depositacionales.

Las frecuencias taxonómicas dan cuenta de restos pertenecientes a guanaco (NISP:71) y huemul (NISP:9), con una mayor representación de los primeros. Los fragmentos diafisiarios, de menor resolución taxonómica, fueron atribuidos al orden artiodactyla, aunque lo más probable es que pertenezcan a guanaco, a juzgar por su mayor representación en el sitio.
En el conjunto también fueron identificados algunos restos óseos (vértebras y porciones de hueso largo) pertenecientes a aves mayores, atribuidos tentativamente al género Chloephaga sp.

$\mathrm{El}$ análisis de la frecuencia esqueletal de guanaco, expone una baja incidencia de factores conservacionales en el conjunto, mostrando especímenes de variada densidad mineral ósea, al igual que una mediana y alta representación de regiones anatómicas, situación que no necesariamente estaría explicada por una conservación diferencial.

Las fusiones óseas determinaron la caza de un guanaco adulto de 35 a 40 meses y una cría de $\leq 10$ meses (Kaufmann 2004) y la presencia de un huemul adulto (cf. Fuentes y Mena 2010).

La incidencia de agentes naturales se verificó en algunos fragmentos de epífisis de guanaco con presencia de mordeduras atribuidas carnívoros, complementariamente al registro de fragmentos óseos de artiodactyla con trazas de ácidos gástricos, producto del paso por el tracto digestivo de algún carnívoro. En adición a rastros derivados de la acción de roedores y eventos de meteorización.

Los rastros antrópicos se identifican en fracturas intencionales y huellas de corte, en restos de artiodactyla y guanaco. Algunas fracturas exponen marcados perimetrales, en fémur proximal y radioulna distal de guanaco, complementariamente a otras de tipo diagonal con claros puntos de impacto, identificadas en la porción anterior y posterior de dos calcáneos, respectivamente. También se identificaron puntos de impacto, lascados y huellas de contragolpe 

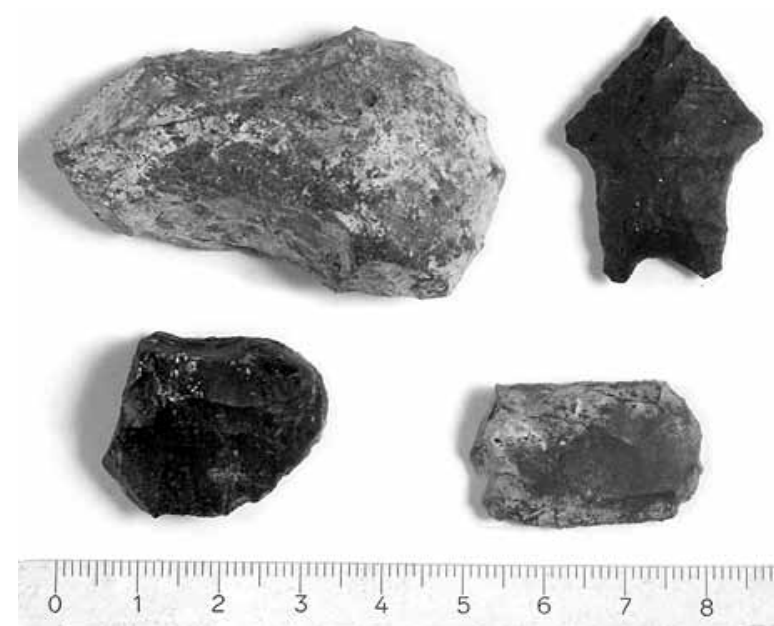

Fig. 5. Artefactos líticos identificados en Alero Gianella.

en algunos especímenes diafisiarios. Mientras que los cortes se encuentran vinculados preferentemente a la extracción de carne, circunstancia que habría sido identificada en el proceso espinoso de tres vértebras lumbares.

Se identificaron fragmentos diafisiarios, correspondientes a artiodactyla, con un largo promedio de $63,98 \mathrm{~mm}$, lo que probablemente sugeriría el aprovechamiento de médula (Mengoni y de Nigris 1999). Por último, se encontraron fracturas de tipo helicoidal, transversal, diagonal y longitudinal, sin rastros evidentes de acción humana (p.e., punto de impacto, contragolpe, etc), aunque el contexto hace presumir un posible origen cultural.

El análisis lítico da cuenta de un total de 228 piezas, las que muestran una amplia representación de la cadena operativa, identificada a partir de la preparación, uso y descarte de instrumentos en el sitio. Las categorías morfofuncionales más abundantes corresponden a derivados de núcleo y desechos de retoque, seguidos por desechos de talla y subproductos o fragmentos aberrantes. Estas cuatro categorías componen el 86,4\% del total de piezas.

En los instrumentos destaca una punta de proyectil, núcleos y raspadores. A estos hallazgos se suman lascas retocadas, una raedera y un percutor. Cabe mencionar que el hallazgo de dos conos de termofractura, más que sugerir un posible tratamiento térmico de las rocas, parece describir el descarte de desechos de producción lítica en un fogón. El sitio expone actividades de extracción y mantención.
Por otro lado, en lo que se refiere a la distribución de materias primas, se advierte una marcada tendencia al uso de andesitas que son presuntamente locales en el valle. La obsidiana, por su parte, presenta escasa frecuencia relativa (15,7\%), contrastando con las frecuencias del valle (cf. Mena et al. 2009). También se registra la utilización de basaltos, que juzgamos como adquiridos en las inmediaciones del sitio. Las materias primas más representadas son las andesitas, con un 45,6\% del total, y variedades de sílices, con un 27,6\%. Lo anterior sugiere que, si bien pareciera que se trata de ocupaciones relacionadas con poblaciones orientales, el sitio habría sido visitado con suficiente regularidad como para conocer los recursos líticos disponibles en sus inmediaciones, apuntando a una mayor residencia en las inmediaciones del lago Gutiérrez.

\section{DISCUSIÓN Y CONCLUSIONES}

El sitio Alero Gianella corresponde a una restringida ocupación por parte de cazadores-recolectores, quienes habrían habitado el sitio durante un período acotado a principios del tercer milenio $\mathrm{AP}$, muy probablemente en visitas estivales, como lo sugiere la presencia de una cría de guanaco.

El alero también mostró rastros de arte rupestre, distinguiendo a este espacio dentro del amplio paredón rocoso que lo alberga, constituyendo así un elemento distintivo en el paisaje. El carácter de las pinturas, posiblemente realizadas por un mismo individuo, su localización en una amplia pared rocosa fuera de la vía natural de circulación y su presunta contemporaneidad con el contexto excavado, sugieren que se trata de un evento aislado, quizás hasta personalizado, sin asociación a ritos de agregación, aunque llama la atención que ésta sea la única evidencia de su tipo conocida en las proximidades del lago Gutiérrez.

El análisis de los restos óseos animales, definió eventos de caza, faenamiento inicial de guanacos y consumo incidental de médula, y en menor medida de huemules y aves, marcando una orientación específica del sitio, apoyada por la presencia de huellas de segmentación y por la ausencia de algunas porciones anatómicas. El análisis lítico da cuenta de diferentes actividades, a juzgar por los instrumentos $y$ artefactos presentes, en contraposición al registro arqueofaunístico. A lo anterior se suma la presencia 
de abundantes materias primas locales, respecto de otras foráneas, dando cuenta del conocimiento de este recurso en el área.

Es posible concluir que Alero Gianella, corresponde al único espacio señalizado con arte rupestre en toda el área del lago Gutiérrez. La favorable ubicación del alero, en el segmento alto del lago, permitió configurar un circuito que articuló con el bosque y la estepa, tal como atestigua la presencia de restos pertenecientes a huemul y guanaco, respectivamente, sectores que por lo demás se ubican en su cabecera (sobre el paredón rocoso que lo alberga) y frente (vista al lago). Paralelamente, la presencia de carcasas incompletas, pertenecientes a guanacos y huemules, nos hace pensar en la hipótesis del traslado o reparto hacia sectores donde las ocupaciones son relativamente sincrónicas y de mayor estabilidad (p.e., áreas de ocupación más amplias, posible uso de toldos, etc), ubicadas hacia la orilla del lago.

La lítica da cuenta de elecciones y procesos reductivos similares a los identificados en trabajos previos para el Chacabuco (Méndez y Blanco 2001; Méndez et al. 2004), constituyendo de este modo un vínculo con Patagonia oriental, definido por la presencia de materias primas alóctonas. Hay que agregar que la diversidad de instrumentos y artefactos líticos, amplía las actividades desarrolladas en el sitio, variando así su funcionalidad "específica", relativa a la caza y procesamiento y consumo inicial de presas, cuestión que muy probablemente está relacionada con la cercanía de posibles campamentos habitacionales, ya mencionados anteriormente, dando cuenta de una particular dinámica microregional (lago Gutiérrez).

A futuro, queda en agenda el desarrollo de prospecciones sistemáticas en los sectores altos del valle, comparables a las desarrolladas en el piso de la cuenca, y excavaciones en el curso bajo, para así comprender mejor el sentido y función de estas ocupaciones.

\section{AGRADECIMIENTOS}

Investigación realizada en el marco del proyecto "Las Ciencias al Servicio del Desarrollo turístico de Aysén, Creando un Centro de Turismo Científico", patrocinado por el Centro de Investigación en Ecosistemas de la Patagonia (CIEP), financiado por INNOVA CORFO, y con el apoyo logístico de
Conservación Patagónica S.A. También queremos agradecer la ayuda otorgada por Trace Gale, Cristian Solis, Emma Faulkner, René Millacura, Jorge Agujera, Alex Triviño y Lilian Henríquez durante las campañas de verano del 2010.

\section{BIBLIOGRAFÍA}

ALTAMIRANO, A. 1983. Guía Osteológica de Cérvidos Andinos. Universidad Nacional Mayor San Marcos, Lima. ASCHERO, C., D. BOZZUTO, M.T. CIVALERO, M. DE NIGRIS, A. DIVRUNO, V. DOLCE, N. FERNANDEZ, L. GONZÁLEZ Y M. SACCHI. 2007. Nuevas evidencias sobre las ocupaciones tempranas en Cerro Casa de Piedra 7. En: Arqueología de Fuego Patagonia; Levantando piedras, desenterrando huesos y ... develando arcanos. pp. 569-576. Ediciones CEQUA, Punta Arenas.

BATE, L. 1970. Las primeras investigaciones sobe arte rupestre de la Patagonia chilena. Anales del Instituto de la Patagonia 1:15-25.

BELARDI, J. Y J. GÓMEZ. 1998. Anatomía económica del huemul (Hippocamelus bisulcus): una contribución a la Interpretación de las evidencias arqueológicas de su aprovechamiento en Patagonia. Anales del Instituto de la Patagonia, Serie Ciencias Humanas 26:195-207.

BINFORD, L. 1981. Bones. Ancient men and modern myths. Academic Press, New York.

1984. Faunal Remains from Klasies River Mouth. Academic Press, New York.

BORRERO, L. 1990. Fuego-Patagonia bone assemblages and problem of communal guanaco hunting. En: Hunters of the Recent Past. Editado por L.B. Davis y B.O.Q. Reeves. Routledge, Londres.

ELKIN, D. 1995. Volume density of South American camelid skeletal part. International Journal of Osteoarchaeology 5:29-37.

FUENTES, F. Y F. MENA. 2010. Estacionalidad y movilidad en cazadores-recolectores. El caso de Cueva Las Guanacas (valle del río Ibáñez, Aisén, Chile). Werkén 13:359-370.

KAUFMANN, C. 2004. La fusión ósea como indicador de edad y estacionalidad en guanaco (Lama guanicoe)". En: Contra viento y marea. Arqueología de la Patagonia, pp.477-487. Instituto Nacional de Antropología y Pensamiento Latinoamericano y Sociedad Argentina de Antropología, Buenos Aires.

LUCERO, V. Y F. MENA. 2001. Arte rupestre del Río Ibáñez: un análisis cuantitativo exploratorio. En: Desde el País de Los Gigantes. Perspectivas arqueológicas en Patagonia, pp. 415-27. Editado por J. B. Belardi, F. Carballo 
y S. Espinosa. Universidad Nacional de la Patagonia Austral, Río Gallegos.

LYMAN, R. 1984. Bone density and differential survivorship of fosil classes. Journal of Anthropological Archaeology 3:259-299.

1994. Vertebrate taphonomy. Cambridge University Press. Oxford.

MENA, F., V. LUCERO, O. REYES, V. TREJO Y H. VELÀSQUEZ. 2001. Cazadores Tardíos en la Cordillera Aisenina: Estudio Comparado de Tres Valles. Informe Final proyecto FONDECYT 1990159, MS.

MENA, F., J. BLANCO Y F. FUENTES. 2009. Informe de investigaciones arqueológicas en valle Chacabuco. Proyecto Las Ciencias al Servicio del Desarrollo turístico de Aysén, Creando un Centro de Turismo Científico. MS.

MÉNDEZ, C. Y J. BLANCO. 2001. Los componentes líticos de los cursos medio y bajo Valle del Chacabuco (Aisén, Chile): Una aproximación exploratoria desde "El Círculo de Piedras" y "El Cuadro del 18". Werken 2:71-82.
MÉNDEZ, C. ; J. BLANCO Y C. QUEMADA. 2004. Aprovechamiento de materias primas líticas en le Alto Chacabuco. Chungara, Revista de Antropología Chilena 36(Vol. Especial):37-47.

MENGONI G. Y M. DE NIGRIS. 1999. Procesamiento de huesos largos de guanaco en Cerro de Los Indios 1 (Santa Cruz). En: Soplando en el viento. Actas de las III Jornadas de Arqueología de la Patagonia, pp. 461475. Neuquén-Buenos Aires.

MUÑOZ, A. S. Y J. B. BELARDI. 1998. El marcado perimetral en los huesos largos de guanaco de Cañadón Leona (colección Junius Bird): implicaciones arqueofaunísticas para Patagonia Meridional. Anales del Instituto de la Patagonia 26: 107-118.

PACHECO, V. ALTAMIRANO, A. Y E. GUERRA. 1986. The osteology of South american camelids. Archaeological reserch tools, Volumen 3. Institute of archaeology. University of California, Los Angeles 3: 1-32. 
